\title{
INFRARED MEASUREMENTS AND ESTIMATION OF TEMPERATURE IN THE RESTRICTIVE SCOPE OF AN INDUSTRIAL CEMENT PLANT
}

\author{
R. Gabriel ${ }^{1}$, S. Keller ${ }^{2}$, J. Matthes ${ }^{3}$, P. Waibel ${ }^{3}$, H. B. Keller ${ }^{3}$, S. Hinz ${ }^{2}$ \\ ${ }^{1}$ ci-tec GmbH, 76137 Karlsruhe, Germany - r.gabriel@ci-tec.de \\ ${ }^{2}$ IPF, Karlsruhe Institute of Technology, 76131 Karlsruhe, Germany - (sina.keller, stefan.hinz)@kit.edu \\ ${ }^{3}$ IAI, Karlsruhe Institute of Technology, 76131 Karlsruhe, Germany - (joerg.matthes, patrick.waibel, hubert.keller) @kit.edu
}

Commission I, WG I/10

KEY WORDS: Infrared measurement, Estimation, Reflectance, Temperature distribution, Clinker cooler, Cement plant, Industrial application

\begin{abstract}
:
In this paper, we describe and evaluate the process of estimating reflectance corrected temperatures based on infrared measurements in the scope of an industrial cement production plant. We overview the underlying cement production phases, as well as the resulting challenges for infrared-based monitoring in such an industrial environment. Our studies are focused particularly on the use of infrared sensors in the clinker cooling process. Using a highly specialized infrared camera $(10.6 \mu \mathrm{m})$, a dataset is obtained capturing the radiation emissions of cement clinker during the clinker cooling process at a cement plant. We briefly turn on the necessity of image preprocessing and then focus on calculating reflectance corrected thermal images for temperature estimation without the use of reference markers or additional instrumentation. This study represents the first usage of infrared camera-based measurements in the clinker cooling process. The main contributions, a recorded dataset and two proposed estimation models including a linear model and a machine learning model with their respective temperature estimations, will provide the basis for the extraction of further process characteristics. Therefore, our contributions will enable scientists as well as process operators to gain new insights about the cement clinker cooling process and to optimize the cement cooling and production process automatically.
\end{abstract}

\section{INTRODUCTION}

Precise data about the two dimensional, spatial distribution of temperature is important in many industrial applications dealing with incineration processes (Matthes et al., 2011; Waibel et al., 2012). Recent developments of specialized infrared cameras provide the possibility of monitoring these burning processes based on image data. Due to the more accurate insight into e.g. the kiln, an improvement of product quality and increase of energy efficiency can be achieved (Vogelbacher et al., 2018). Especially in the cement industry, as one major branch of industry depending on efficient and smooth burning processes, the monitoring of the kiln temperatures gains considerable benefits.

Although the monitoring of the kiln temperature works quite well and infrared thermography is widely used in various other fields ( e.g. Nilsson (1991); Kaplani (2012); Grinzato et al. (2002); McCafferty et al. (1998); Sanchez et al. (2011)), solely few studies have been conducted focusing on the temperature distribution of the clinker cooler operation (e.g. Smith and Burke (1998)). None of these studies have used infrared camera-based measurements. Because the cooling step decisively affects the quality of the produced cement clinker, we think that the use of infrared technology in the clinker cooler is an essential tool for process monitoring.

In the context of the clinker cooler operation, which is the focus of this contribution, four crucial challenges appear:

1. achieving a homogeneous and fast cooling of the clinker,

2. circumvention of snowman,

3. prevention of red river and

4. boosting of energy recuperation from secondary and tertiary air.
In order to meet these challenges, the entire clinker cooling process needs to be considered, monitored and modeled. This starts by understanding the sensory recordings as well as the determination of characteristic parameters and ends by encroaching on the process control.

To address the stated challenges, we propose the usage of an infrared-based camera. Using the recorded infrared image data combined with intelligent algorithms, the clinker cooling process can be monitored comprehensively. Thus, its temperature distribution can be evaluated in detail. The proposed approach fosters innovative feedback by glancing insights to the clinker cooling process, allowing direct verification of changes impeded on the process.

The main objective of the presented work is to estimate the temperature distribution of the cement clinker in the cooler. Although an infrared camera directly yields radiation information, these measurements are always a combination of emission, reflectance, and transmission effects. Basing downstream information acquisition and decision steps on such measurements is therefore errorprone and has to be avoided.

Furthermore, we have to deal with several restrictions when conducting infrared measurements at the cement clinker cooler. The first restriction arises due to the setup and pysical construction of the clinker cooler, resulting in very limited positioning possiblities for the camera and therefore unfavorable field of view. The second restriction arises from the prevalant temperature conditions and rought environment, prohibiting the use of additional instrumentation. The fact that the process runs 24/7 fundamentally requires an online evaluation and demands a robust as well 
as reliable approach. Such restrictions are softened in other thermographic applications where infrared measuring techniques are applied (cf. Iwaszczuk et al. (2011, 2013)). Additionally, the absence of reference data impede the evaluation and requires a sophisticated understanding of the underlying process. We rely on a dataset which was recorded at a cement plant during the common operation exclusively for this study.

The main contributions of this paper are:

1. measurement campaign for acquisition of an infrared dataset,

2. extraction of first process parameters,

3 . generation of reflectance adjusted temperature measurements without the knowledge of any reference data.

First, we establish a general understanding of the cement production process in Section 2. Subsequently, we explain the inherent challenges of infrared-based measurements in this specific industrial application. A description of the used sensor and recorded dataset is given in Section 3. Necessary preprocessing steps and extracted process characteristics are listed in Section 4. Our proposed models for estimating the temperature distribution of the cement clinker cooler based on highly reflecting infrared data is introduced in Section 5. In Section 6, we present the derived results of our proposed models before ending with our conclusions and an outlook in Section 7.

\section{CEMENT PRODUCTION}

The process of cement production ${ }^{1}$ is divided into four main steps which are described briefly in the following subsections:

1. Extraction and processing of raw material (Section 2.1)

2. Burning of clinker (Section 2.2)

3. Cooling of clinker (Section 2.3)

4. Grinding, blending and packaging (Section 2.4)

Calcium oxide $(\mathrm{CaO})$ and silicon dioxide $\left(\mathrm{SiO}_{2}\right)$ are the main raw ingredients for clinker production. Further necessary elements are alumina $\left(\mathrm{AI}_{2} \mathrm{O}_{2}\right)$ and iron oxide $\left(\mathrm{Fe}_{2} \mathrm{O}_{2}\right)$. Raw materials like limestone, chalk and clay, or the naturally occurring combination limestone marl, provide the majority of these constituent parts. Depending on the available commodities, pure limestone, iron ore, or sand are added to balance the chemical composition. Other possible source materials comprise lime mud, used foundry sand and fly ash (VDZ, 2016).

\subsection{Raw material extraction and processing}

Raw material mining mostly is conducted by controlled blasting. The coarse materials are ground into smaller fragments and layered in mixing beds, assuring a consistent product composition. The mixed materials combined with possible additives are fed into a raw mill, in which the materials are ground. The ground materials are well mixed and dried with hot air obtained from the burning process in the kiln and/or from the clinker cooler process.

The now ready-mixed raw meal is dried further via grate or cyclone preheaters. In the latter case an additional precalcinator is often used, utilizing exhaust air from the kiln and additional fuels to start the endothermic deacidification reaction. This early deacidification accounts for shorter rotary kilns and the utilization of secondary fuels.

\footnotetext{
${ }^{1}$ We only describe the production of cement in a rotary kiln with a grate cooler.
}

\subsection{Rotary kiln}

The preheated raw meal is fed into the rotary kiln, a tube lined with refractory brickwork with diameters of up to $6 \mathrm{~m}$ and a length between $10 \mathrm{~m}$ to $200 \mathrm{~m}$. The kiln is mounted with a $3^{\circ}$ to $4^{\circ}$ incline and rotates between 1.3 and 3.5 times per minute. Opposite from the infeed point (at the lower end of the kiln) a burner is installed. The raw meal is slowly transported from the infeed to the outfeed side of the kiln, taking about $20 \mathrm{~min}$ to $40 \mathrm{~min}$. When arriving at the sintering zone the raw meal reaches temperatures around $1450{ }^{\circ} \mathrm{C}$ and becomes the so-called clinker. The surrounding gas reaches temperatures up to $2000^{\circ} \mathrm{C}$ and resides in the kiln for only $2 \mathrm{~s}$ to $4 \mathrm{~s}$. A detailed description of rotary kilns is given in Boateng (2008).

\subsection{Clinker cooler}

After reaching the end of the rotary kiln, the clinker falls from the rotary kiln into the cooler. Therefore a chute-like construction is used to guide the falling clinker onto the stationary part of the clinker cooler. The stationary part is angled around $15^{\circ}$ and uses air, blown through slots in the grate, to cool the clinker. To aid with equal distribution of the clinker, the air can usually be directed from one side to the other. After leaving the gravity feed stationary part, the clinker moves onto the dynamic part of the grate, which has a decreased inclination of approx. $3^{\circ}$. The dynamic part of the cooler also uses air which is blown through from below to cool the clinker. Usually, the air flow can be regulated for different sections of the dynamic cooler. To ensure the transport of the clinker, the dynamic grate is comprised of beams that move back and forth in an alternating order, thus, continuously pushing the clinker towards the end of the cooler. The heated exhaust air is mainly used as secondary air in the kiln and in the form of tertiary air in a possible precalcination step. Some additional air might be used in drying processes further upstream. The cooled clinker should leave the cooler with temperatures between $80^{\circ} \mathrm{C}$ to $200^{\circ} \mathrm{C}$. A very important factor determining the characteristics of the clinker is the cooling rate: a faster cooling results in finer tricalcium silicate $\left(\mathrm{Ca}_{3} \mathrm{SiO}_{5}\right)$ which leads to higher initial hardness of the produced cement and can also reduce energy demand during the grinding (Zement-Taschenbuch, 2002). Two common error patterns arising in the operation of a grate clinker cooler are the following:

\section{Red river}

The formation of so-called red rivers resembles a lava-like stream of hot clinker (Gagnon, 1997). This phenomenon occurs when cooling air cannot penetrate fine and closely packed clinker. Hence, the affected areas reach high temperatures resulting in a fluidization of the clinker which than moves quicker than the rest of the bed. Thus, these parts have even less time to cool down. Based on the rotation direction of the kiln ${ }^{2}$ and the accompanying segregation, fine clinker tends to accumulate on one side of the cooler.

Red rivers cause damage to grate plates and produce frequent downtimes (Wolff et al., 1995). Moreover, they also result in inferior cement quality and less efficient energy recuperation.

\section{Snowman}

A snowman is an accumulation of fine incandescent dust, that is layered like stalagmites, mainly inside the chute area of the

\footnotetext{
${ }^{2}$ In our case the rotation direction is counterclockwise when looking from the clinker cooler side.
} 


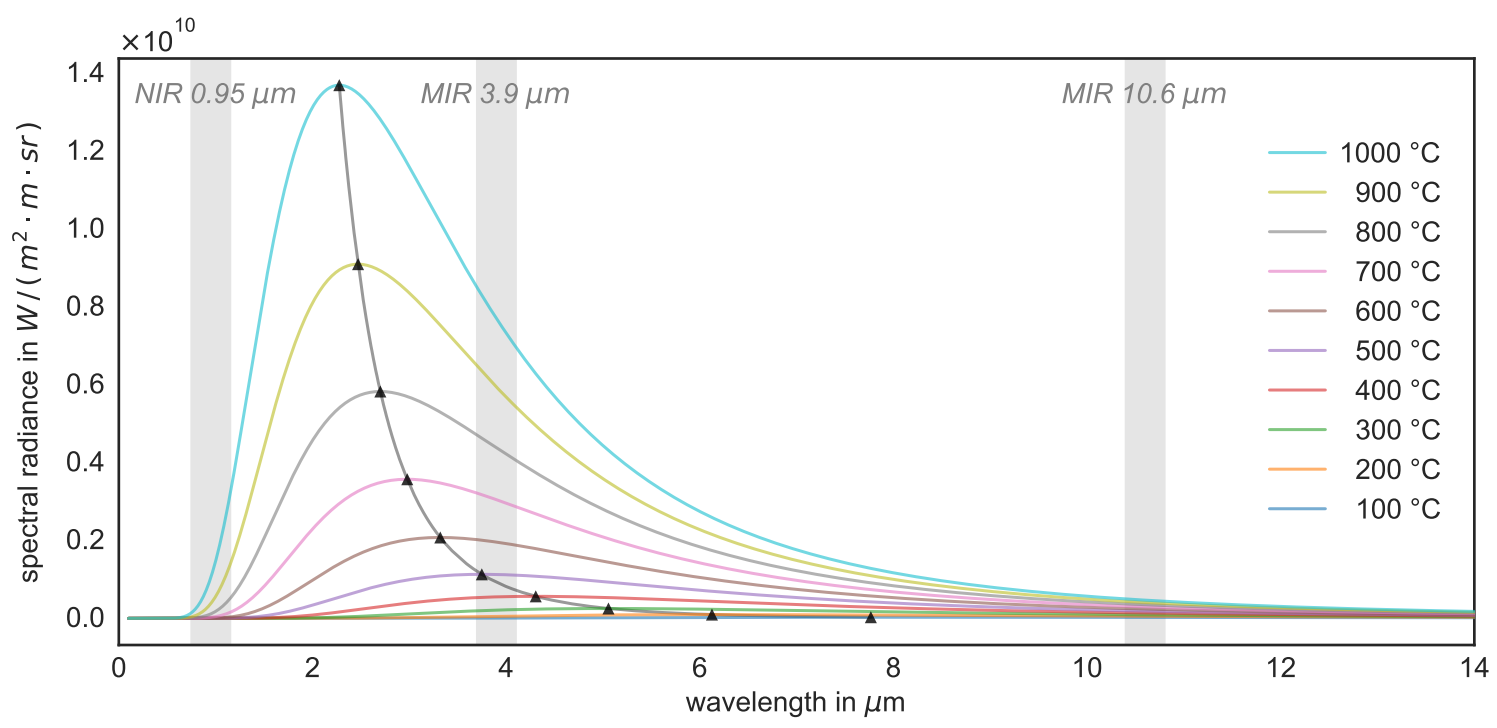

Figure 1. Black-body radiation of different temperatures.

clinker cooler (Gagnon, 1997). It can be caused by e.g. very dusty clinker, a distorted flame in the kiln or blowback between cooler and kiln. The result is a sticky like wet snow behavior of the clinker (Meyer and Wolter, 1997), leading to an inhomogeneous clinker bed and additional aerodynamic challenges.

\subsection{Grinding and blending}

After the cooling step the resulting clinker has formed nodules, usually between $3 \mathrm{~mm}$ to $50 \mathrm{~mm}$ in diameter. Clinker, gypsum, granulated slag and other additives are ground and mixed according to the specific demands of the produced cement. Based on growing requirements on the produced cement, separated grinding of all the ingredients to their desired grain size before mixing becomes more and more common.

\section{INFRARED SENSOR AND DATASET}

The operating conditions for all equipment used in the cement industry are quite harsh since cement dust is everywhere. The dust flows into every opening. In addition, the production process requires extremely high temperatures of up to $2000{ }^{\circ} \mathrm{C}$ and installation positions are limited by physical accessibility. The used equipment does not only need to withstand these conditions permanently, but also function reliably. In this context, every interruption in the process decreases the product quality or results in a useless product and is therefore quite costly.

Infrared cameras capable of withstanding these kinds of conditions are rare, and fairly expensive. Therefore, some limitations in comparison with other, state of the art, imaging technology have to be mastered. Most infrared cameras available for high temperature industrial applications operate in one of three spectral regions: $0.95 \mu \mathrm{m}, 3.9 \mu \mathrm{m}$ and $10.6 \mu \mathrm{m}$. According to DIN 5031 the first ones reside within the near infrared (NIR) band, while the other two are representatives of the mid infrared (MIR) band. Black-body radiation is quite strong in the spectral range around $3.9 \mu \mathrm{m}$ for the expected temperatures in a clinker cooler (cf. Figure 1). Yet, available cameras are incapable of measuring temperatures below $600{ }^{\circ} \mathrm{C}$, only allowing information retrieval from the beginning of the cooling process and obstructing possible insight to the later process steps, where expected temperatures are well below $600^{\circ} \mathrm{C}$. The temperatures at the end of the clinker cooler for example, range from $80^{\circ} \mathrm{C}$ to $200^{\circ} \mathrm{C}$. Black-body radiation in the spectral range around $0.95 \mu \mathrm{m}$ is not powerful enough at temperatures below $700{ }^{\circ} \mathrm{C}$ either, hence a camera utilizing the $10.6 \mu \mathrm{m}$ band was selected.

The specific camera is a PYROINC $380 L F / 50 H Z / 74 X 52$ operating at $10.6 \mu \mathrm{m} \pm 0.25 \mu \mathrm{m}^{3}$ with a maximum frame rate of $50 \mathrm{~Hz}$ and a sensor resolution of $384 \times 288$ pixels. The temperature measuring capabilities range from $0{ }^{\circ} \mathrm{C}$ to $2040{ }^{\circ} \mathrm{C}$. Figure $2 \mathrm{a}$ shows an exemplary image obtained by this camera. For more information about the camera see DIAS infrared (2018). The camera captures radiance values, which are converted to temperature values in software. This conversion is inherently non-linear, however for the utilized temperature range in the clinker cooler a linear relation can be assumed for ease in processing.

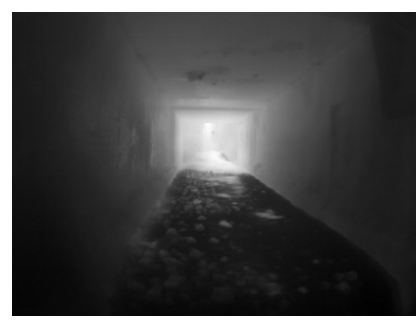

(a) Sample image

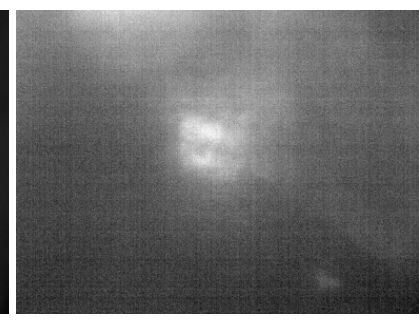

(b) Poor visibility
Figure 2. View into a clinker cooler.

The used dataset was recorded under fully operating conditions in a cement plant and consists of five $10.6 \mu \mathrm{m}$-MIR, three $0.95 \mu \mathrm{m}$ NIR, and three recordings in the visual band. The recordings are of varying length, between one minute and one hour. The recordings were obtained on a single day by inserting the camera through a particularly prepared opening in the wall at the end of the clinker cooler, which is accessible from a small platform. Water cooling and purge air were used to cool the camera electronics and to keep the lens free of any contamination.

\footnotetext{
${ }^{3}$ with an $5 \%$ cut-on/cut-off
} 


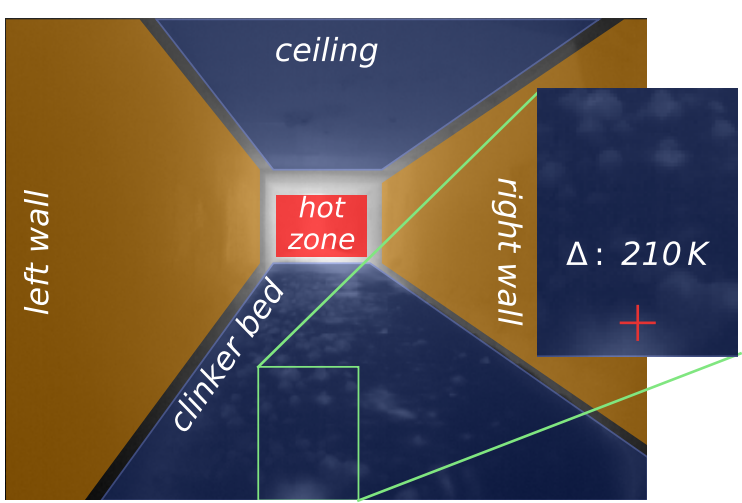

Figure 3. Main zones of the clinker cooler. The red cross marks point of maximum frame-to-frame temperature difference.

In this paper, we solely rely on the recordings of the $10.6 \mu \mathrm{m}-$ camera, primarily from a 51 min sequence captured with $25 \mathrm{~Hz}$.

\subsection{Challenges for image acquisition and processing}

Obviously, the spatial resolution is barely still sufficient, especially when considering the viewpoint. The smallest structures at the infeed side of the cooler, that theoretically can be resolved by the camera, are in the order of $10 \mathrm{~cm}$. This is aggravated by the fact, that the already limited possibilities to influence the process are decreasing as the clinker bed moves in the direction towards the camera. Although the effective spatial resolution increases, adjustments of the cooler have less time to influence the clinker.

Poor visibility states an additional challenge due to particles in the air (cf. Figure 2b). These circumstances result typically from (a) the usage of air-cannons, which are employed to disintegrate snowman (cf. Section 2.3 and other conglomerates, or (b) as a result of bigger cakings that have fallen down from the kiln and raise some finer clinker particles. Dust arising from the grinder right under the outfeed side of the cooler has an additional negative effect on the visibility conditions throughout the whole cooler. The dust is then being pulled through the entire cooler by active process air, influencing all steps of the clinker burning process. While information concerning the deployment of air-cannons can be gathered from the process control system, the duration of the poor visibility condition is incomprehensible. For other occurring circumstances, no additional information is available beforehand. Thus, the system faces these circumstances on its own (cf. Section 4 for the applied solutions).

Concerning infrared temperature measurements in general, an issue is the missing information on the specific values of emissivity, reflectivity, and transmittance with respect to the recorded scene. By contrast with other applications of infrared measurements, these values are unknown and change simultaneously. In the example given in Figure 3 the temperature indicated by the red cross in the zoomed area changes by $210 \mathrm{~K}$ from one frame to the following ${ }^{4}$, while the scene remains stable.

\section{PREPROCESSING}

Before tackling the overall objective of estimating a real temperature distribution, several preprocessing steps are executed.

\footnotetext{
${ }^{4}$ In this case, a frame length is $1 / 25 \mathrm{~s}$
}

These steps contain image assessment, temporal filtering, projective transformation as well as segmentation and retransformation. When selecting the preprocessing methods and estimation models, we have to consider that reliability is the major demand in a $24 / 7$ industrial application.

\subsection{Quality assessment of images}

The first step in the image processing chain is the assessment of the image quality. We calculate different statistical measures of image regions and compare them against one another. If, for example, the mean value and its deviation for different zones, located in the clinker bed and on the wall, are very similar, this indicates an extremely high dust content, which makes information extraction in further image processing steps virtually impossible. Thereupon, such images are discarded in subsequent steps.

\subsection{Temporal filtering}

The next step takes advantage of the relatively slow process in the clinker cooler. Based on the selected image data of the previous step, we calculate the mean value for each pixel (cf. Equation (1))

$$
I_{f(t)}(x, y)=\frac{1}{|I|} \cdot \sum_{i=1}^{|I|} I_{i}(x, y)
$$

$$
\text { where } \quad \begin{aligned}
& I_{f(t)}=\text { filtered image at time } \mathrm{t} \\
& I_{t}=\text { image at time } \mathrm{t} \\
& |I|=\text { number of valid images between } t-1 \text { and } t \\
& x, y=\text { image coordinates }
\end{aligned}
$$

The temporal filtering effectively eliminates short-term perturbations and measurement noise of the data.

\subsection{Projective transformation}

In order to enable later processing steps to calculate meaningful characteristic values concerning the clinker bed, we use a projective transformation to generate a virtual top view (cf. Figure $4 \mathrm{a}$ ). Note, that the shown transformation is horizontally stretched, the real aspect ratio is close to 1:6. The transformation parameters are obtained from CAD drawings related to the clinker cooler. The homogeneous projective transformation matrix is determined via a normalized direct linear transform and the resulting temperature-pixels are determined via bilinear interpolation. Based on the limited positioning possibilities of the camera, the resulting inequality in effective resolution has to be accepted. The virtual top view enables us to generate profound features based on the geometric model of the clinker cooler, e.g. we can calculate the size of certain structures (e.g. clinker nodules see Section 4.4) in the bed, allowing for process insights.

\subsection{Segmentation and back transformation}

One use of the transformed image is the segmentation of clinker nodules in the clinker bed. Clinker nodules are agglomerates of various sizes, resembling rocks on a sandy beach. These nodules tend to store heat for longer periods, encompassing very hot material, thereby preventing a homogeneous cooling process and possibly even causing damage to the grinder. Figure $4 \mathrm{~b}$ shows an example of segmented nodules. In this image, the increasing quality of the segmentation is clearly visible as the clinker moves 


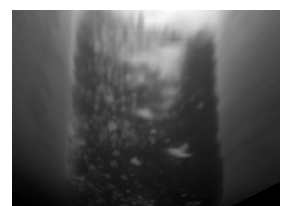

(a) Projective transformation of clinker bed

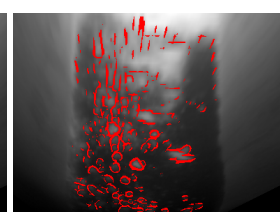

(b) Segmentation in transformed image

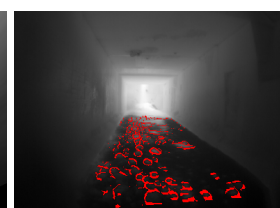

(c) Back projection of segmentation
Figure 4. Projective transformation and exemplary application.

towards the camera. This information is useful for operating the cooler, as it not only holds information on the size of clinker nodules, but also their spatial distribution.

The back-projected segmentation (cf. Figure 4c) clearly reveals that bigger nodules tend to live on the left side. This leads to rather compact, fine-grained clinker on the right side of the cooler, which is a culprit for the emergence of red rivers.

\section{MODEL OF REFLECTANCE CORRECTION}

Dealing with reflectance is an elaborate task at hand. Especially when emissivity, reflectivity, and transmittance are not only unknown, but change over time and the introduction of calibration surfaces or other means of calibration are highly impractical. Reliable temperature measurements are important indicators for product quality, an essential information for process control and the ability to decrease downtimes. It is also critical for red river detection, where streams of hot clinker pervade the clinker bed (cf. Section 2.3). Thus, retrieving such reliable measurements has a high priority in industrial applications.

In the following sections, absolute temperatures are denoted in Celsius $\left({ }^{\circ} \mathrm{C}\right)$ while temperature differences are denoted in Kelvin $(\mathrm{K})$. Note that temperature differences are given as relative differences and therefore negative values are possible, although negative Kelvin are physically impossible.

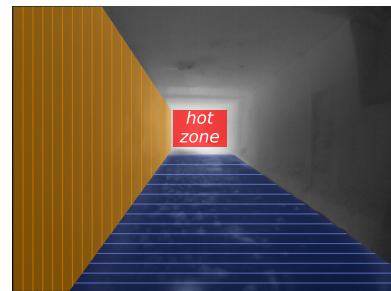

(a) Partitions of the left wall and clinker bed zones

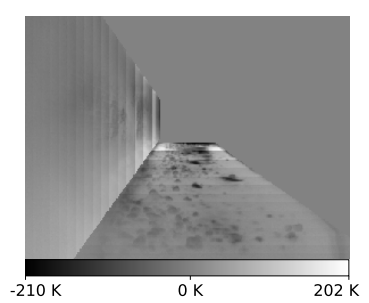

(b) Estimation of linear equation less measured temperatures
Figure 5. Partitioning and results of the linear model.

\subsection{Linear Model}

Since the measured radiation is a combination of the real emitted energy at the measured point and the additive reflective component, our first approach is to solve the system of linear equations (cf. Equation (2)) for every defined partition in the cooler. Note, that we use the measured temperature values instead of the radiation values, since the mapping can be assumed linear as a first

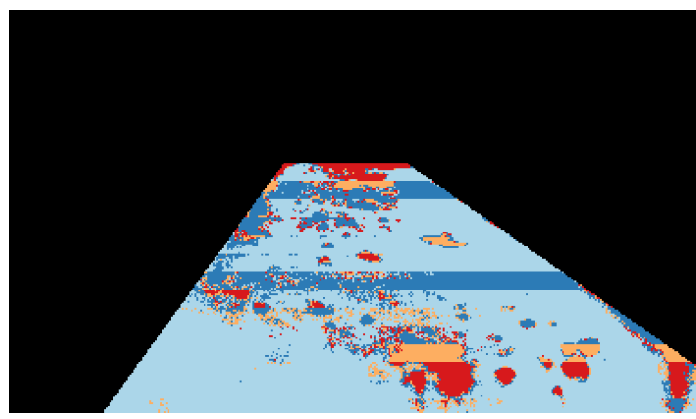

Figure 6. Example of found clusters in the clinker bed zones. Note that each zone has its individual five clusters, although they are shown with the same colors

approximation.

$$
\left[\begin{array}{cc}
1 & \bar{\Theta}_{\text {hotzone }}(1) \\
\vdots & \vdots \\
1 & \bar{\Theta}_{\text {hotzone }}(n)
\end{array}\right] \cdot\left[\begin{array}{c}
\Theta_{\text {rad }} \\
\alpha_{\text {refl }}
\end{array}\right]=\left[\begin{array}{c}
\Theta_{\text {Meas }}(1) \\
\vdots \\
\Theta_{\text {Meas }}(n)
\end{array}\right]
$$

where

$$
\begin{array}{ll}
\bar{\Theta}_{\text {hotzone }}(t)= & \text { mean temperature of the hot zone } \\
& \text { at time } \mathrm{t} \\
= & \text { radiation based temperature } \\
\Theta_{\text {rad }} & \text { share of temperature reading } \\
\alpha_{\text {refl }} & \text { originating of reflectance } \\
\Theta_{\text {Meas }}(t)= & \text { temperature readings at time } \mathrm{t}
\end{array}
$$

Figure 5a shows a visualization of the partitions for the left wall and the clinker bed. The resulting solutions, namely $\Theta_{\text {rad }}$ and $\alpha_{\text {refl }}$, are stable over large spans of training time. When looking at the difference between the estimated and the measured temperatures however (cf. Figure 5b), it is striking that estimations for hot regions are generally too low and estimations for cold regions are too high. This is caused by the influence of high reflectance areas which overflow into the other areas. Liable for this behavior is the use of the average temperature value as a representation for the whole partition. Consequently, when looking at the more static wall segments, the estimations match more closely. The correlation even increases when considering the last third of the wall where the average difference amounts to only $6.7 \mathrm{~K}$.

In order to impede the problem of overflow, we propose a more targeted model for reflectance adjusted temperature measurements. We utilize the following properties of the process to achieve this goal:

1. slow locomotion of the clinker

2. lethargy of build structures against temperature changes

3. stable temperatures over a long time horizon

4. insensitiveness to reflections of hot zone temperatures

The first property is expressed by the $20 \mathrm{~min}$ dwell time the clinker resides on the dynamic part of the cooler. It allows for extended filtering over time and the extraction of characteristics of certain zones in the clinker bed. We therefore keep the partitioning of the dynamic part of the clinker bed and the left wall as shown in Figure 5a. However, we additionally execute a kmeans clustering, dividing each segment into five clusters (cf. Figure 6). The five clusters were empirically chosen, for giving the best cost/benefit relationship. Note that the clusters of individual zones are independent of one another, yet are shown 


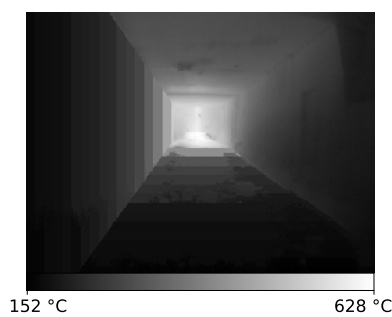

(a) Estimation of cluster-based linear equation

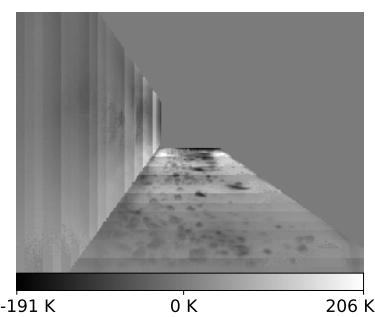

(b) Estimation of cluster-based linear equation less measured temperatures
Figure 7. Results of cluster-based linear equation system.

with the same colors. Hence, we retrieve fine-grained information about the clinker distribution in each segment.

The next step is to train individual systems of equations for each partition-cluster combination. The estimation results are shown as an overlay in Figure 7a. Similarly as before, Figure 7b illustrates the difference between the estimated and measured temperatures. Overall, the estimated temperatures tend to be slightly higher when using clusters compared with simply averaged segments, while positive and negative deviations are close to symmetric with $\pm 45 \mathrm{~K}$

The availability of $\Theta_{\text {rad }}$ and $\alpha_{\text {refl }}$ as separate entities enable us to also generate estimations based solely on hot zone reflections or on emission (cf. Figure 8).

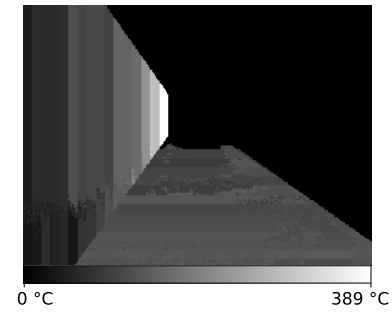

(a) Estimation of temperatures based soley on emission

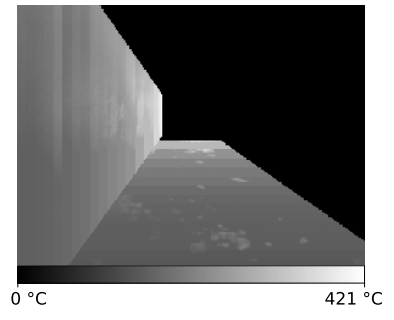

(b) Estimation of temperatures based soley on reflectance
Figure 8. Separation of estimated emission and estimated reflectance.

\subsection{Ensemble tree bagger model}

For the last model we train bootstrap-aggregated (bagged) decision trees as a means of regression between the hot zone temperature and the different clusters in each of the zones. The model utilizes quantile regression forests (Meinshausen, 2006). We apply the median quartile as our predictor to receive fitting temperature prognoses. We chose the bagged decision trees approach to reduce overfitting and improve generalization, while keeping a fast, flexible and easy to use approach. The training step was performed with 100 bags of trees for every partition-cluster combination.

New data is assigned to the nearest cluster. An estimation based on the regression result and the most recent hot zone temperature is made (cf. Equation (3)). In order to generate the adjusted temperature measurements, we calculate:

$$
\Theta_{\text {adj }}(x, y)=\Theta(x, y)-\Theta_{\text {pred }}(x, y)+\Theta_{\text {mean }}(x, y)
$$

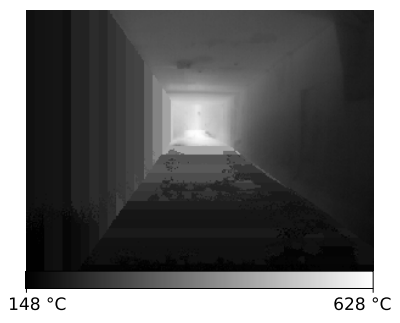

(a) Estimation of temperatures with cluster-based tree bagger

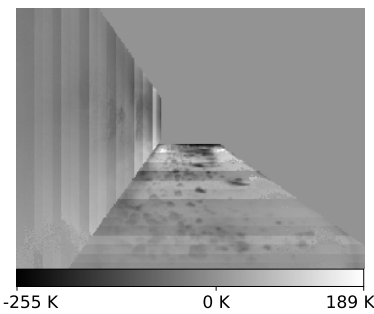

) Estimation of temperatures with cluster-based tree bagger less measured temperatures
Figure 9. Results of cluster-based tree bagger.

where

$$
\begin{aligned}
& \Theta_{\text {adj }}=\text { adjusted temperatures } \\
& \Theta \quad=\text { measured temperatures } \\
& \Theta_{\text {pred }}=\text { estimated temperatures } \\
& x, y \quad=\text { image coordinates } \in \text { zones }
\end{aligned}
$$

This is a valid approach, since average temperature in the zones differ only by a few $\mathrm{K}$, whether observed for short or long periods of time. The resulting image of adjusted temperatures is slightly blurred (Gaussian blur, $\sigma=0.5$ ) to reduce artifacts at the transitions between clusters (as visible in Figure 6).

As before, the adjusted thermal image is combined with the infrared temperature measurements, resulting in e.g. Figure 9.

\section{EVALUATION OF THE RESULTS}

Since no reference data is available, evaluation is an extremely challenging task. For the evaluation of the results, we consider feasibility as well as profiles of the estimated temperatures. Figure 11 shows the resulting adjusted thermographic images. The points with maximum frame-to-frame $e^{5}$ temperature difference in the clinker bed are highlighted. All model estimations reduce the amount of temperature deviation considerably. This is also illustrated by the fact, that maximum average frame-to-frame temperature difference is at least halved for all models (cf. Table 1). The model using a system of linear equations for each zone-cluster combination clearly performs best, followed by the model using a system of linear equations for every zone. This indicates, that using a system of linear equations produces reliable reflectance adjusted thermographic measurements. When looking at the estimated temperatures however, both models produce much too low temperatures at the infeed side of the cooler. Realistic temperatures on the beginning of the dynamic grate are above $300^{\circ} \mathrm{C}$, but the estimations are often below $150^{\circ} \mathrm{C}$. On the other hand estimations for the zones near the end of the cooler are close to $100^{\circ} \mathrm{C}$, which is rather realistic.

\begin{tabular}{lc}
\hline Model & $\overline{\Delta \Theta}$ in $\mathrm{K}$ \\
\hline Raw Measurement $(R M)$ & 16.86 \\
Linear equation whole zones $\left(L E_{W Z}\right)$ & 6.22 \\
Linear equation cluster $\left(L E_{C}\right)$ & 4.51 \\
Tree bagger cluster $\left(T B_{C}\right)$ & 8.38 \\
\hline
\end{tabular}

Table 1. Maximum average frame-to-frame temperature difference.

\footnotetext{
${ }^{5}$ Note: One frame represents the filtered information for one second.
} 


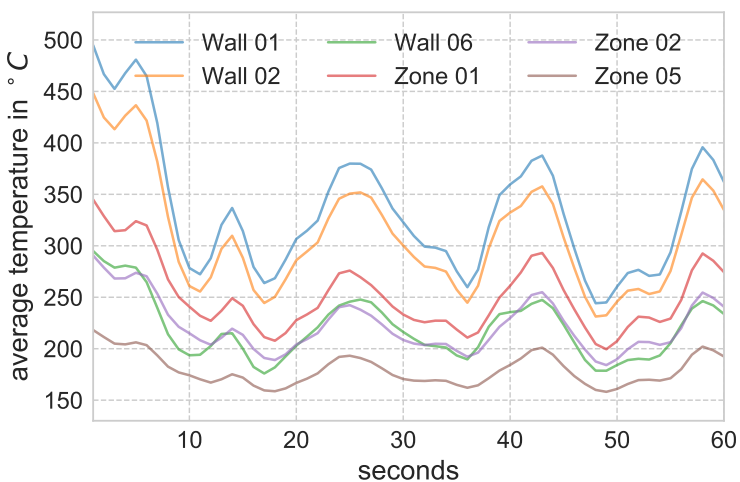

(a) Average temperature of zones from raw measurements $(R M)$.

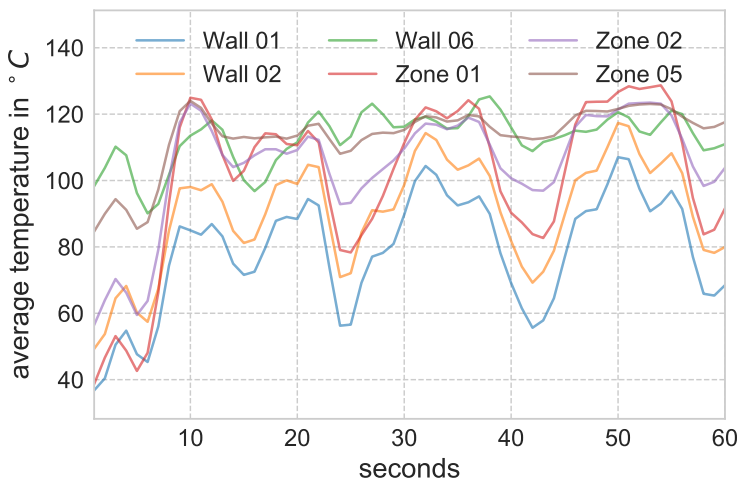

(c) Average temperature of zones based on Linear equations using cluster $\left(L E_{C}\right)$

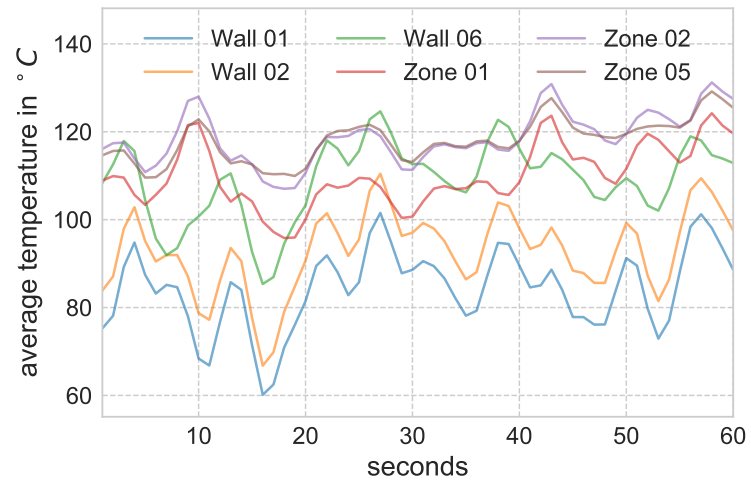

(b) Average temperature of zones based on Linear equation using whole zones $\left(L E_{W Z}\right)$.

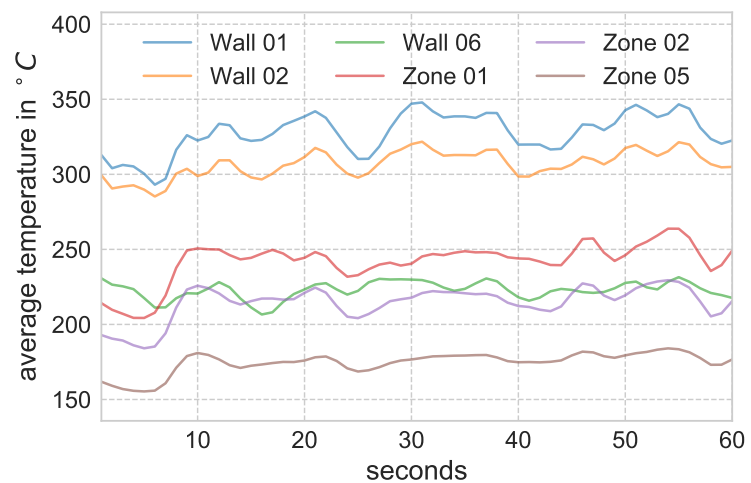

(d) Average temperature of zones based on Tree bagger cluster $\left(T B_{C}\right)$ estimations

Figure 10. The average temperatures in selected zones during a 1 min sequence. Note the different temperature scales.

For the further evaluation, we show the average temperature ${ }^{6}$ of a few examplary zones for each of the proposed models in Figure 10. Note, that $1 \mathrm{~min}$ sequences are shown for presentation reasons, while the evaluation is based on longer time spans of up to one hour each. Clinker bed and wall zones with lower numbers are closer to the hot zone. Table 2 shows the maximum differences in the average zone temperatures, again all three models reduce the fluctuations in the temperature profiles. As expected, the model using the system of linear equations for entire zones produces the lowest variations, since the estimated temperatures resemble the learned mean value for this zone. Notably, the tree bagger model produces similar results while using clustered input data. Additionally, it also produces the lowest frame-to-frame temperature difference (Figure 11d). This suggests, that it is the most appropriate model for obtaining a reflectance adjusted temperature measurement.

When looking at the evaluation results, both models deliver good results, but also exhibit weaknesses. At least to some extent these weaknesses could be eliminated by combining the results of the different models, for example, based on the geometric location of the estimation areas in the cooler. Another possible enhancement would be to incorporate process knowledge into the estimation models such as feasible temperature ranges or information acquired in the rotary kiln.

\footnotetext{
${ }^{6}$ Gaussian smoothed with $\sigma=1$
}

\begin{tabular}{ccccc}
\hline Zone & $\begin{array}{c}\text { RM } \\
\text { in K }\end{array}$ & $\begin{array}{c}\mathrm{LE}_{w Z} \\
\text { in K }\end{array}$ & $\begin{array}{c}\mathrm{LE}_{\mathrm{C}} \\
\text { in K }\end{array}$ & $\begin{array}{c}\mathrm{TB}_{\mathrm{C}} \\
\text { in K }\end{array}$ \\
\hline Wall $_{1}$ & 250 & 41 & 70 & 54 \\
Wall $_{2}$ & 217 & 43 & 68 & 36 \\
Wall $_{5}$ & 119 & 39 & 35 & 24 \\
$\mathrm{CB}_{1}$ & 145 & 28 & 90 & 59 \\
$\mathrm{CB}_{2}$ & 106 & 24 & 67 & 45 \\
$\mathrm{CB}_{5}$ & 60 & 19 & 39 & 28 \\
\hline
\end{tabular}

Table 2. Maximum average zone temperature difference for Raw Measurements $(R M)$, Linear equation whole zones $\left(L E_{W Z}\right)$, Linear equation cluster $\left(L E_{C}\right)$, Tree bagger cluster $\left(T B_{C}\right)$ in $\mathrm{K}$. Clinker bed $\left(C B_{1,2,5}\right)$ and wall zones $\left(W_{a l l} l_{1,2,5}\right)$ with lower numbers are closer to the hot zone.

\section{CONCLUSION AND OUTLOOK}

The employed means to measure temperatures in clinker coolers range from conventional temperature probes (mainly for predictive maintenance applications) over line scanners to CCDSensors (Smith and Burke, 1998; Wen-Ming et al., 2006). Using infrared cameras however, is a novel idea. To our knowledge this is the first realization of such a system using an infrared camera, therefore fundamental research has to be conducted.

We have proposed the basic steps comprising the process of cement production and illustrate, how industrial requirements and challenges affect the application of infrared cameras as thermal 

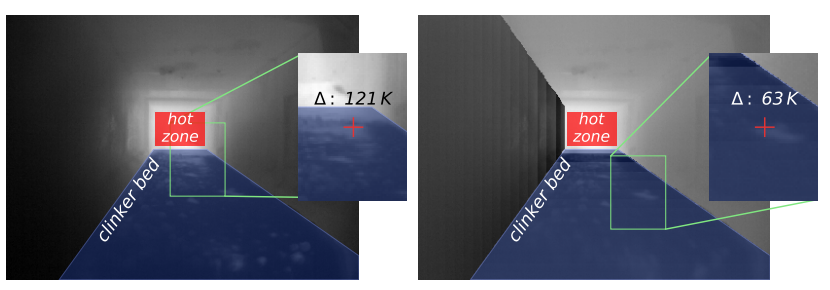

(a) Raw measurement

(b) Linear equations whole zones

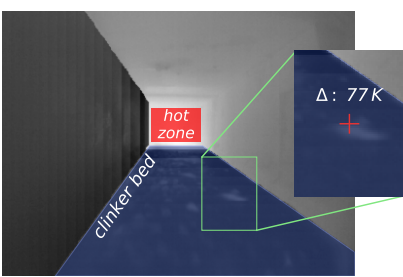

(c) Linear equation with clusters

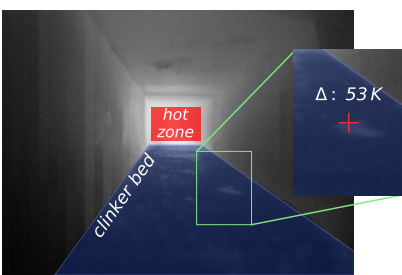

(d) Tree bagger

Figure 11. The maximum temperature difference from one frame to the other in a 1 minute sequence

sensors. Additionally, we presented our measured dataset, as well as some basic preprocessing and feature extraction methods. Most importantly, we presented two different models to acquire reflectance adjusted thermal measurements from within a clinker cooler. Theoretically, these models would be able to run robustly in a 24/7 industrial application. The results are promising, indicating that infrared-based thermography could become an indispensable tool for clinker cooler operators. Then, it reveals the potential of widening the optimization portfolio of the cement industry.

In future research we would like to expand on the proposed approaches. This includes the evaluation of the proposed methods in a 24/7 live operating plant. Another interesting approach is the combination of both methods, where robustness and reliability could possibly be improved. The acquisition of spectral pyrometer data as reference data is a further task, we are planning to carry out. Based on this data, enhanced machine learning models could be applied to estimate the temperature distribution of the cement clinker while cooling. The usage of conventional temperature measuring equipment, i.e. thermocouples could lead to an externally referenced calibration. Another intention is to generate an artificial evaluation dataset via ray-tracing and a 3D-model of a clinker cooler.

\section{References}

Boateng, A. A., 2008. Rotary kilns: Transport phenomena and transport processes. Elsevier/Butterworth-Heinemann, Amsterdam.

DIAS infrared, 2018. Pyroview 380l. http://www. dias-infrared.de/pdf/pyroview3801_eng_mail.pdf (01 April 2018).

DIN 5031, 1984. Strahlungsphysik im optischen Bereich und Lichttechnik; Benennung der Wellenlängenbereiche.

Gagnon, D., 1997. Upgrading a clinker cooler. IKN Klinker Inlet Distribution System ("KIDS"). IEEE Transactions on Industry Applications 33(1), pp. 126-134.

Grinzato, E., Bressan, C., Marinetti, S., Bison, P. and Bonacina, C., 2002. Monitoring of the Scrovegni Chapel by IR thermography: Giotto at infrared. Infrared Physics \& Technology 43(35), pp. 165-169.

Iwaszczuk, D., Helmholz, P., Belton, D. and Stilla, U., 2013. Model-to-image registration and automatic texture mapping using a video sequence taken by a mini UAV. Proceedings of the International Archives of the Photogrammetry, Remote Sensing and Spatial Information Sciences, Hannover, Germany pp. 21-24.

Iwaszczuk, D., Hoegner, L. and Stilla, U., 2011. Detection of windows in building textures from airborne and terrestrial infrared image sequences. Archiwum Fotogrametrii, Kartografii i Teledetekcji Vol. 22, pp. 215-225.

Kaplani, E., 2012. Detection of Degradation Effects in FieldAged c-Si Solar Cells through IR Thermography and Digital Image Processing. International Journal of Photoenergy 2012(1), pp. 1-11.

Matthes, J., Waibel, P. and Keller, H. B., 2011. A new infrared camera-based technology for the optimization of the Waelz process for zinc recycling. Minerals Engineering 24(8), pp. 944-949.

McCafferty, D. J., Moncrieff, J. B., Taylor, I. R. and Boddie, G. F., 1998. The use of IR thermography to measure the radiative temperature and heat loss of a barn owl (Tyto alba). Journal of Thermal Biology 23(5), pp. 311-318.

Meinshausen, N., 2006. Quantile regression forests. The Journal of Machine Learning Research 7, pp. 983-999.

Meyer, H. and Wolter, A., 1997. To improve the availability of grate coolers. In: 1997 IEEE Cement Industry Technical Conference Record XXXIX, I E E E, Piscataway, pp. 227-254.

Nilsson, H. E., 1991. Hand-held radiometry and IRthermography of plant diseases in field plot experiments $\dagger$. International Journal of Remote Sensing 12(3), pp. 545-557.

Sanchez, J. M., French, A. N., Mira, M., Hunsaker, D. J., Thorp, K. R., Valor, E. and Caselles, V., 2011. Thermal Infrared Emissivity Dependence on Soil Moisture in Field Conditions. 49(11), pp. 4652-4659.

Smith, C. and Burke, K. J., 1998. Cooler scanner technology [cement plant control]. In: 1998 IEEE Cement Industry Technical Conference, I E E E, Piscataway, pp. 391-402.

VDZ, 2016. Umweltdaten der deutschen Zementindustrie 2016.

Vogelbacher, M., Waibel, P., Matthes, J. and Keller, H. B., 2018. Image-Based Characterization of Alternative Fuel Combustion With Multifuel Burners. IEEE Transactions on Industrial Informatics 14(2), pp. 588-597.

Waibel, P., Vogelbacher, M., Matthes, J. and Keller, H. B., 2012. Infrared Camera-based Detection and Analysis of Barrels in Rotary Kilns for Waste Incineration. In: Proceedings of the 2012 International Conference on Quantitative InfraRed Thermography, QIRT Council.

Wen-Ming, Z., Bin, L. and Hai, Z., 2006. Study on measurement system of cement cooler clinker's temperature field. In: 2006 2nd IEEE/ASME International Conference on Mechatronics and Embedded Systems and Applications, pp. 1-4.

Wolff, R., Alesi, S. and Miller, S., 1995. Applying modern technology in a step by step approach to improve the capacity and efficiency of a preheater/calciner kiln system. In: 1995 IEEE Cement Industry Technical Conference XXXVII, IEEE, Piscataway, pp. 225-248.

Zement-Taschenbuch, 2002. Zement-Taschenbuch, Vol. 50, 50. aufl. edn, Verlag Bau + Technik, Düsseldorf. 